\title{
Meditation during a pandemic
}

Dr Anju Bhatia, a speciality paediatric dentist and clinical teacher, describes the meditation sessions that have been held at King's College Hospital during the COVID-19 crisis.

ince 30 March to date [21 May] we at King's College Hospital have been holding four to six meditation sessions each day from Monday to Friday. Our sessions begin at 8 am and go on throughout the day; a few evenings a week the final session is at $8 \mathrm{pm}$.

The sessions are for our COVID critical care staff and A\&E, paediatric, Liver ITU and dental staff. We also have open sessions each day for all staff from every other part of the hospital. Consultants, clinical leads, medical and dental staff, matrons, nurses, key workers, cleaning, auxiliary and security staff have all attended the sessions.

All attendees have benefitted from the meditation sessions and the testimonials have been amazing and profound.

Following the Twin Hearts Meditation by MCKS, each session is 30 minutes; we begin with gentle exercise, deep breathing, visualisation and erasing negative emotion techniques and then we have a guided meditation with love for oneself, the Earth

\section{LDC Conference moves online}

This year's LDC Conference is being held online on Saturday 25 July (Chair is Leah Farrell).

The day will incorporate much of what is core to Conference: the discussion of motions; the opportunity to hear about the latest developments in dental politics and debate with key figures in the profession; and networking with colleagues from across the UK - but this year in a virtual format. For more information visit https://www.ldcuk.org/. and humanity at its centre. We have also developed a self-care book for our staff to use at home, encouraging them to continue with the self-care techniques taught to them in the sessions.

The sessions have been introduced to help keep our staff emotionally resilient and balanced at this difficult time, where some are facing their worst fears.

We have also raised enough money through one of our charities to give each meditator a lavender and salt bath soak to take home at night to relax the body after wearing PPE for a 12-hour shift. Again, this has been very well received by our staff and we are promoting wellbeing of the mind and the body.

The impact of these sessions has been so very profound that we are now in the process of developing research programmes to more closely evaluate the benefit of them. This will include all of our staff, and we are developing a research programme for our patients too on the benefits these sessions may bring to them.

I personally have practised these

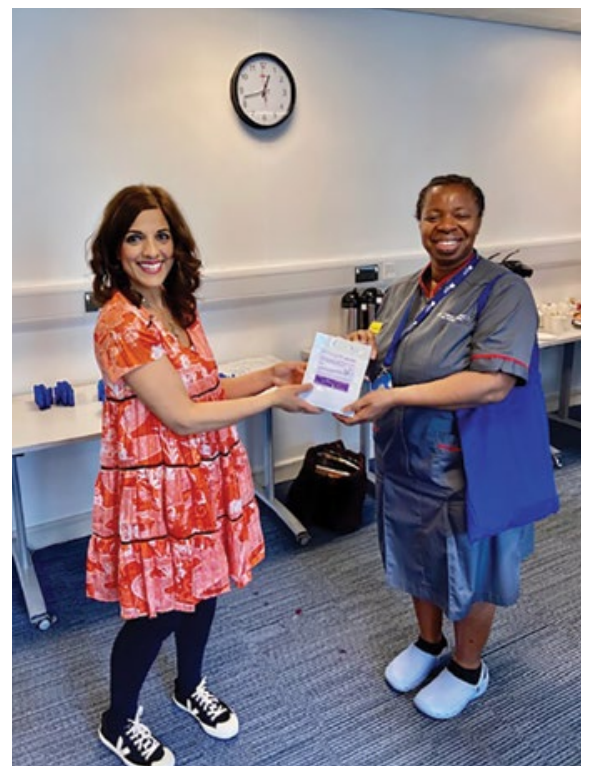

Anju (left) presenting a staff member with a lavender and salt bath soak

meditation techniques following a very difficult period in my personal life, and they rapidly helped me to recover, emotionally rebalance and start to live again. My team at King's supported me through this time and when we began to incorporate meditative practice into our daily lives at the hospital, all those involved felt that their stress levels improved.

My wellbeing work at King's has grown organically at a rapid rate, helping many of our staff through this period.

\section{How do you feel about orthodontic retainers in general practice?}

Orthodontic treatment in the UK continues to be highly requested by both children and adults. This treatment involves a time and financial commitment from the patients and often their parents. Included within almost all orthodontic consent will be long term or indefinite retention, to maintain the patient's teeth in their aligned position. Retention strategies may involve bonded or removable appliances and for the most part the general dental practitioner takes on the responsibility of monitoring these appliances.

We are interested in how these appliances are monitored and what happens when problems are encountered.

We are asking UK-based BDA members to complete a short five-minute survey, the findings of which will be published in the $B D J$. The implications of this may inform future commissioning and remuneration.

The survey is open to all UK-based dental practitioners and is available at https:// meded.onlinesurveys.ac.uk/retainer-studysurvey until 31 August 2020.

The researchers involved would be most grateful for your participation. 